International Journal of Pure and Applied Mathematics

Volume 112 No. 1 2017, 47-55

ISSN: 1311-8080 (printed version); ISSN: 1314-3395 (on-line version)

url: http://www.ijpam.eu

doi: 10.12732 /ijpam.v112i1.3

\title{
ON LATTICE ORDERED SOFT GROUPS
}

\author{
L. Vijayalakshmi ${ }^{1}$, J. Vimala $^{2} \S$ \\ ${ }^{1,2}$ Department of Mathematics \\ Alagappa University \\ Karaikudi, Tamilnadu, INDIA
}

\begin{abstract}
Molodsov in [15] initiated soft set theory, which can be used as a mathematical tool for dealing uncertainties. In this paper the concept of lattice ordered soft group is introduced and some of its properties and operations have been derived.
\end{abstract}

AMS Subject Classification: 03G10, 06B05

Key Words: soft sets, soft groups, lattice ordered soft sets, lattice ordered soft groups

\section{Introduction}

Molodsov in [15], who was the first researcher initiated the theory of soft sets in 1999. This theory is mainly used for dealing the problems with uncertainties like fuzzy set theory[4], rough set theory[4], probability theory and many. P.K. Maji et.al[14] discussed some operations of soft sets. Irfan Ali et.al [3] and Aslihan Sezgin and Akin Osman Atagun[2] proved different operations on soft sets. Fundamentals of soft set theory were briefly discussed with some examples by Onyeozili and Gwary[21]. Aktas and Cagman introduced the notion of soft sets and developed the soft group theory in [1]. Also, Muhammad Aslam et.al [16] extended the operations of soft group and some of its properties were verified. Further, Xia yin and Zuhua Liao[23] introduced the new concept of soft subgroups and normal soft subgroups. Muhammad Irfan Ali introdued the notion of lattice (anti-lattice) ordered soft sets in [5]. Some properties of

\begin{tabular}{lr}
\hline Received: & May 31,2016 \\
Revised: & October 18,2016 \\
Published: & January 17,2017 \\
${ }^{\S}$ Correspondence author
\end{tabular}

(c) 2017 Academic Publications, Ltd. url: www.acadpubl.eu 
lattice(anti-lattice) ordered soft sets and their algebraic structures were investigated. Lattice theory has been applied to all kinds of fields. Lattice theory, a branch of mathematics was introduced by Garett Birkhoff[10]. R. Natarajan, and J. Vimala $[19,20]$ have introduced standard and distributive $l$-ideal in a commutative lattice ordered group. Also the triple representation for a super modular semilattice was introduced by J. Vimala [24 ] and the role of fuzzy l-ideals in a commutative lattice ordered group[25] was also established by J. Vimala and P. Bharathi.

In this paper, we define the notion of the lattice ordered soft group and have studied some of its properties and operations.

This paper has been organized as follows: In Section 2, some preliminary definitions and examples have been outlined. In Section 3, the definition of lattice ordered soft group is given and few operations have been analysed.

\section{Preliminaries}

In this section, we give some basic definitions and results of lattices, soft sets and soft groups.

A poset $(L, \leq)$ is said to form a lattice if for every $a, b \in L, S u p\{a, b\}=a \vee b$ and $\operatorname{Inf}\{a, b\}=a \wedge b$ exist in $L$. Every chain is a lattice. Dual of a lattice is a lattice. Product of two lattices is a lattice. A latttice $L$ is called a complete lattice if every non-empty subset of $L$ has its Sup and Inf in $L$. Dual of a complete lattice is complete. A non-empty subset $S$ of a lattice $L$ is called a sublattice of $L$ if $a, b \in S \Rightarrow a \vee b, a \wedge b \in S$.

Next some defintions about soft sets and soft groups are discussed.

Definition 2.1. ([16]) Let $U$ be an initial universe set and $E$ a set of parameters with respect to $U$. Let $P(U)$ denote the power set of $U$ and $A \subseteq E$. A pair $(F, A)$ is called a soft set over $U$, where $F$ is a mapping given by $F$ : $A \rightarrow P(U)$. In other words, a soft set $(F, A)$ over $U$ is a parameterized family of subsets of $U$. For $e \in A, F(e)$ may be considered as the set of $e$-elements or $e$-approximate elements of the soft set $(F, A)$.

Definition 2.2. ([16]) Let $(F, A)$ and $(H, B)$ be two soft sets over common universe $U$, we say that $(H, B)$ is said to be a soft subset of $(F, A)$ if

1. $A \subseteq B$ and

2. $F(e) \subseteq H(e)$ for all $e \in A$.

We write $(H, B) \tilde{C}(F, A)$, and $(H, B)$ is said to be a soft super set of $(F, A)$ if $(F, A)$ is a soft subset of $(H, B)$. We denote it by $(H, B) \tilde{\supset}(F, A)$ 
Definition 2.3. ([16]) Two soft sets $(F, A)$ and $(H, B)$ over a common universe $U$ are said to be soft equal if $(F, A)$ is a soft subset of $(H, B)$ and $(H, B)$ is a soft subset of $(F, A)$.

Definition 2.4. ([16]) A soft set $(F, A)$ over $U$ is said to be a Null soft set denoted by $\Phi$ if for all $e \in A, F(e)=\emptyset$.

Definition 2.5. ([16]) A soft set $(F, A)$ over $U$ is said to be a Absolute soft set denoted by $\widetilde{A}$ if for all $e \in A, F(e)=U$.

Definition 2.6. ([15]) Let $(F, A)$ and $(G, B)$ be two soft sets over a common universe $U$. Then the union of $(F, A)$ and $(G, B)$ denoted $(F, A) \tilde{\cup}(G, B)$ is a soft set $(H, C)$, where $C=A \cup B$ and for all $e \in C$,

$$
H(e)= \begin{cases}F(e), & \text { if } e \in A-B \\ G(e), & \text { if } e \in B-A \\ F(e) \cup G(e), & \text { if } e \in A \cap B\end{cases}
$$

Definition 2.7. ([15]) Let $(F, A)$ and $(G, B)$ be two soft sets over a common universe $U$. Then the intersection of $(F, A)$ and $(G, B)$ denoted $(F, A) \tilde{\cap}(G, B)$ is a soft set $(H, C)$, where $C=A \cap B$ and $\forall e \in C, H(e)=F(e)$ or $G(e)$ (as both are same set).

Definition 2.8. ([15]) Let $(F, A)$ and $(G, B)$ be two soft sets over a common universe $U$ such that $A \cap B \neq \emptyset$. Then their restricted intersection is denoted by $(F, A) \cap(G, B)=(H, C)$, where $(\mathrm{H}, \mathrm{C})$ is defined as $H(c)=$ $F(c) \cap G(c)$, for all $c \in C$.

Definition 2.9. ([15]) The extended intersection of two soft sets $(F, A)$ and $(G, B)$ over a common inverse $U$ is the soft set $(H, C)$, where $C=A \cup B$, and for all $e \in C, H(e)$ is defined as

$$
H(e)= \begin{cases}F(e), & \text { if } e \in A-B \\ G(e), & \text { if } e \in B-A \\ F(e) \cap G(e), & \text { if } e \in A \cap B\end{cases}
$$

We write $(F, A) \sqcap_{E}(G, B)=(H, C)$.

Definition 2.10. ([15]) The restricted union $(H, C)$ of two $\operatorname{soft} \operatorname{sets}(F, A)$ and $(G, B)$ over the common universe $U$ is defined as the soft set $(H, C)=$ $(F, A) \cup_{R}(G, B)$ where $C=A \cap B$ and $H(c)=F(c) \cup G(c)$, for all $c \in C$.

Definition 2.11. ([15]) Let $(F, A)$ and $(G, B)$ be two soft sets over a common universe $U$. Then extended union $(H, C)$ of two soft sets $(F, A)$ and 
$(G, B)$ is denoted as the soft set $(H, C)=(F, A) \cup_{E}(G, B)$, where $C=A \cup B$, and $H$ is defined as

$$
H(e)= \begin{cases}F(e), & \text { if } e \in A-B \\ G(e), & \text { if } e \in B-A \\ F(e) \cup G(e), & \text { if } e \in A \cap B\end{cases}
$$

We write $(F, A) \cup_{E}(G, B)=(H, C)$.

Definition 2.12. ([23]) Let $(F, A)$ and $(G, B)$ be two soft sets over a common universe $U$. Then the basic union of $(F, A)$ and $(G, B)$ defined as the soft set $(H, C)=(F, A) \bigvee(G, B)$ where $C=A \times B$, and $H(a, b)=F(a) \cup G(b)$, for all $(a, b) \in A \times B$.

Definition 2.13. ([23]) Let $(F, A)$ and $(G, B)$ be two soft sets over a common universe $U$. Then the basic intersection of $(F, A)$ and $(G, B)$ defined as the soft set $(H, C)=(F, A) \bigwedge(G, B)$ where $C=A \times B$, and $H(a, b)=$ $F(a) \cap G(b)$, for all $(a, b) \in A \times B$.

Definition 2.14. ([1]) Let $G$ be a group and $E$ be a set of parameters. For $A \subseteq E$, the pair $(F, A)$ is called a soft group over $G$ if and only if $F(a) \leq G$ for all $a \in A$, where $F$ is a mapping of $A$ into the set of all subsets of $G$. That is, A soft group is a parameterized family of subgroups.

Definition 2.15. ([1]) Let $(F, A)$ and $(H, K)$ be two soft groups over $G$. Then $(H, K)$ is a soft subgroup of $(F, A)$ written as $(H, K) \tilde{<}(F, A)$, if

1. $K \subseteq A$

2. $H(x) \leq F(x)$ for all $x \in K$.

Two soft groups are equal if $(F, A) \tilde{<}(H, K)$ and $(H, K) \tilde{<}(F, A)$.

Definition 2.16. ([5]) If $U$ is an initial universe, then $P(U)$ is a bounded lattice and the set of parameters $E$, is also a lattice with respect to certain binary operations (or) partial order and $A \subseteq E$. A soft set $(F, A)$ is called a lattice ordered soft set if for the mapping $F: A \rightarrow P(U), x \leq y$ implies $F(x) \subseteq F(y)$, for all $x, y \in A \subseteq E$.

\section{Lattice Ordered Soft Groups}

In this section, we introduce the notion of lattice ordered soft group and its operations. 
Definition 3.1. Let $G$ be a group and $P(G)$ be the power set of $G$. Let $E$ be the set of parameters(lattice), $A \subseteq E$. Then a soft set $(F, A)$ is said to be a lattice ordered soft group if for the mapping $F: A \rightarrow P(G)$,

1. $F(a)$ is a subgroup of $G$, for all $a \in A \cdot[1]$

2. If $a \vee b, a \wedge b \in A$ then $\operatorname{Sup}\{F(a), F(b)\}$ and $\operatorname{Inf}\{F(a), F(b)\}$ exist in $(F, A)$, for all $a, b \in A$.

Note: Throughout this section, $F(a) \vee F(b)$ and $F(a) \wedge F(b)$ are used for $\operatorname{Sup}\{F(a), F(b)\}$ and $\operatorname{Inf}\{F(a), F(b)\}$ respectively, also $\vee$ denotes $\cup$ and $\wedge$ denotes $\cap$.

Note: L denotes the set of all lattice ordered soft groups.

Example 3.2. Assume that $G=Z$ is the universal group under the operation of addition and $E=N$ is the set of all Natural numbers. Then $(F, N)$ is defined by $F(n)=\left\{z_{n}: n \in N, z \in Z\right\}$ is a lattice ordered soft group over Z.

Example 3.3. Assume that $G=Z$ is the universal group under the operation of addition and $E=P$ is the set of all Prime numbers. Then $(F, P)$ is defined by $F(p)=\{p z: p \in P, z \in Z\}$ is not a lattice ordered soft group over Z.

Proposition 3.4. In a lattice ordered soft group $(F, A), F(a) \vee F(b)$ and $F(a) \wedge F(b)$ exist in $(F, A)$ if and only if $F(a) \subseteq F(b)$ (or) $F(b) \subseteq F(a)$, for all $a, b, \in A$.

Proposition 3.5. Let $(F, A) \in L$, for all $a, b, c \in A$ and $F(a), F(b), F(c) \in$ $(F, A)$. Then the following results hold

Idempotency: $F(a) \vee F(a)=F(a)$ and $F(a) \wedge F(a)=F(a)$.

Commutativity: $F(a) \vee F(b)=F(b) \vee F(a)$ and $F(a) \wedge F(a)=F(b) \wedge F(a)$.

Associativity: $F(a) \vee[F(b) \vee F(c)]=[F(a) \vee F(b)] \vee F(c)$ and $F(a) \wedge[F(b) \wedge$ $F(c)]=[F(a) \wedge F(b)] \wedge F(c)$.

Absorption: $F(a) \vee[F(a) \wedge F(b)]=F(a)$ and $F(a) \wedge[F(a) \vee F(b)]=F(a)$.

Proposition 3.6. A lattice ordered soft group is a poset.

Proposition 3.7. Let $(F, A),(G, B) \in L . \quad A \subseteq B$ if and only if $(F, A) \tilde{\subset}(G, B)$, for all $A, B \subseteq E$.

Proof. Assume $A \subseteq B$. Let $a_{1}, a_{2} \in A$ implies $a_{1}, a_{2} \in B$. Therefore, $a_{1} \vee a_{2}, a_{1} \wedge a_{2} \in A$ implies $a_{1} \vee a_{2}, a_{1} \wedge a_{2} \in B$. Then we get $F\left(a_{1}\right) \vee F\left(a_{2}\right), F\left(a_{1}\right) \wedge$ $F\left(a_{2}\right)$ exist in $(F, A)$ implies $F\left(a_{1}\right) \vee F\left(a_{2}\right), F\left(a_{1}\right) \wedge F\left(a_{2}\right)$ exist in $(G, B)$. Hence $(F, A) \tilde{\subset}(G, B)$. 
Conversely, assume $(F, A) \tilde{\subset}(G, B)$.

Let $F\left(a_{1}\right) \vee F\left(a_{2}\right), F\left(a_{1}\right) \wedge F\left(a_{2}\right) \in(F, A)$, for all $a_{1}, a_{2} \in A$. Then $F\left(a_{1}\right) \vee$ $F\left(a_{2}\right), F\left(a_{1}\right) \wedge F\left(a_{2}\right) \in(G, B)$, for all $a_{1}, a_{2} \in A$. Since $A$ and $B$ are lattices, $a_{1} \vee a_{2}, a_{1} \wedge a_{2}$ exist in $A$ implies $a_{1} \vee a_{2}, a_{1} \wedge a_{2} \in B$. Hence $A \subseteq B$.

Proposition 3.8. Let $(F, A),(G, B) \in L$. Then $(F, A) \tilde{\cup}(G, B)=(G, B)$ and $(F, A) \tilde{\cap}(G, B)=(F, A)$ if and only if $(F, A) \tilde{\subset}(G, B)$, for all $A, B \subseteq E$.

Proof. Let $(F, A) \tilde{\cup}(G, B)=(G, B)$ and $(F, A) \tilde{\cap}(G, B)=(F, A)$. Suppose for all $a_{1}, a_{2} \in A, F\left(a_{1}\right) \vee F\left(a_{2}\right), F\left(a_{1}\right) \wedge F\left(a_{2}\right) \in(F, A)$. $\Rightarrow$ for all $a_{1}, a_{2} \in A$, $F\left(a_{1}\right) \vee F\left(a_{2}\right), F\left(a_{1}\right) \wedge F\left(a_{2}\right) \in(F, A) \tilde{\cup}(G, B)$.

$\Rightarrow$ for all $a_{1}, a_{2} \in A, F\left(a_{1}\right) \vee F\left(a_{2}\right), F\left(a_{1}\right) \wedge F\left(a_{2}\right) \in(G, B)$. Hence $(F, A) \tilde{\subset}(G, B)$. Conversely, Suppose $(F, A) \tilde{\subset}(G, B)$. Let $(F, A) \tilde{\cup}(G, B)=$ $(H, C)$, where $C=A \cup B$.

Let $a_{1}, a_{2} \in A \cup B, F\left(a_{1}\right) \vee F\left(a_{2}\right), F\left(a_{1}\right) \wedge F\left(a_{2}\right) \in(F, A) \tilde{\cup}(G, B)$.

Then $F\left(a_{1}\right) \vee F\left(a_{2}\right), F\left(a_{1}\right) \wedge F\left(a_{2}\right)$ exist in $(H, C), \forall a_{1}, a_{2} \in A \cup B$. By our assumption, $(F, A) \tilde{\subset}(G, B)$ if and only if $A \subseteq B$ implies $C=A \cup B=B$. Then $F\left(a_{1}\right) \vee F\left(a_{2}\right), F\left(a_{1}\right) \wedge F\left(a_{2}\right)$ exist in $(H, A \cup B)$. Therefore for all $c \in$ $C, H(c)=G(c)$. That is $(H, C)=(G, B)$. Hence $F\left(a_{1}\right) \vee F\left(a_{2}\right), F\left(a_{1}\right) \wedge F\left(a_{2}\right)$ exist in $(G, B)$. Therefore $(F, A) \tilde{\cup}(G, B) \subseteq(G, B)$.

Let $\forall b_{1}, b_{2} \in B, G\left(b_{1}\right) \vee G\left(b_{2}\right), G\left(b_{1}\right) \wedge G\left(b_{2}\right)$ exist in $(G, B)$.

Then $\forall b_{1}, b_{2} \in B, G\left(b_{1}\right) \vee G\left(b_{2}\right), G\left(b_{1}\right) \wedge G\left(b_{2}\right)$ exist in $(F, A) \tilde{\cup}(G, B)$. Therefore $(G, B) \subseteq(F, A) \tilde{\cup}(G, B)$. Thus $(F, A) \tilde{\cup}(G, B)=(G, B)$. Similarly we can prove $(F, A) \tilde{\cap}(G, B)=(F, A)$.

Theorem 3.9. Let $(F, A),(G, B) \in L$, its extended union $(F, A) \cup_{E}$ $(G, B) \in L$ if $(F, A) \tilde{\subset}(G, B)($ or $)(G, B) \tilde{\subset}(F, A)$.

Proof. Let $(H, C)=(F, A) \cup_{E}(G, B)$, where $C=A \cup B$. Suppose $(F, A) \tilde{\subset}(G, B)$. By the proposition 3.7, we have $A \subseteq B$ implies that $C=$ $A \cup B=B$. Then $(H, C)=(H, A \cup B)=(G, B)$ implies $\forall c \in C, H(c)=G(c)$. Since $(G, B)$ is a lattice ordered soft group, $(H, C)$ is also a lattice ordered soft group. Similarly if $(G, B) \tilde{\subset}(F, A)$, we get $(H, C)=(F, A)$ is a lattice ordered soft group.

Theorem 3.10. If $(F, A),(H, B) \in L$, then its restricted intersection $(F, A) \cap(H, B) \in L$.

Proof. Let $(K, C)=(F, A) \cap(H, B)$, where $C=A \cap B \neq \emptyset$.

Let $F(a) \vee F(b), F(a) \wedge F(b)$ exist in $(F, A)$, then $F(a) \subseteq F(b)$ or $F(b) \subseteq$ $F(a)$, for all $a, b \in A$ and let $H(a) \vee H(b), H(a) \wedge H(b)$ exist in $(H, B)$, then $H(a) \subseteq H(b)$ or $H(b) \subseteq H(a)$, for all $a, b \in B$. Now for all $a, b \in A \cap B$, we have $F(a) \cap H(a) \subseteq F(b) \cap H(b)$ (or) $F(b) \cap H(b) \subseteq F(a) \cap H(a)$.

$\Rightarrow K(a) \subseteq K(b)$ (or) $K(b) \subseteq K(a)$, for all $a, b \in C=A \cap B$. Therefore 
$K(a) \vee K(b), K(a) \wedge K(b)$ exist in $(K, C)$. Hence $(K, C)$ is a lattice ordered soft group.

Theorem 3.11. If $(F, A),(G, B) \in L$, then its restricted union $(F, A) \cup_{R}$ $(G, B) \in L$.

Proof. Let $(H, C)=(F, A) \cup_{R}(G, B)$, where $C=A \cap B \neq \emptyset$. Let $F(a) \vee F(b), F(a) \wedge F(b)$ exist in $(F, A)$, then $F(a) \subseteq F(b)$ or $F(b) \subseteq F(a)$ for all $a, b \in A$ and let $G(a) \vee G(b), G(a) \wedge G(b)$ exist in $(G, B)$, then $G(a) \subseteq G(b)$ or $G(b) \subseteq G(a)$ for all $a, b \in B$. Now for all $a, b \in A \cap B$, we have $F(a) \cup G(a) \subseteq$ $F(b) \cup G(b)$ (or) $F(b) \cup G(b) \subseteq F(a) \cup G(a)$.

$\Rightarrow H(a) \subseteq H(b)$ (or) $H(b) \subseteq H(a)$, for all $a, b \in C=A \cap B$. Therefore $H(a) \vee H(b), H(a) \wedge H(b)$ exist in $(H, C)$. Hence $(H, C)$ is a lattice ordered soft group.

Theorem 3.12. If $(F, A),(H, B) \in L$, then its extended intersection $(F, A) \sqcap_{E}(H, B) \in L$.

Proof. Let $(K, C)=(F, A) \sqcap_{E}(H, B)$, where $C=A \cup B$.

Let for all $a_{1}, a_{2} \in A, F\left(a_{1}\right) \vee F\left(a_{2}\right), F\left(a_{1}\right) \wedge F\left(a_{2}\right)$ exist in $(F, A)$ and for all $b_{1}, b_{2} \in B, H\left(b_{1}\right) \vee H\left(b_{2}\right), H\left(b_{1}\right) \wedge H\left(b_{2}\right)$ exist in $(H, B)$. Suppose $A \cap$ $B=\emptyset$, if $\forall c \in A-B, K(c)=F(c)$ and if $\forall c \in B-A, K(c)=H(c)$. Since $(F, A)$ and $(H, B)$ are lattice ordered soft groups, then for all $c_{1}, c_{2} \in$ $C, K\left(c_{1}\right) \vee K\left(c_{2}\right), K\left(c_{1}\right) \wedge K\left(c_{2}\right)$ exist in $(K, C)$. Suppose $A \cap B \neq \emptyset$, if for all $a_{1}, a_{2} \in A-B, F\left(a_{1}\right) \vee F\left(a_{2}\right), F\left(a_{1}\right) \wedge F\left(a_{2}\right)$ exist in $(F, A)$, if $b_{1}, b_{2} \in$ $B-A, H\left(b_{1}\right) \vee H\left(b_{2}\right), H\left(b_{1}\right) \wedge H\left(b_{2}\right)$ exist in $(H, B)$, and if for all $c_{1}, c_{2} \in A \cap B$, then from the restricted intersection theorem, $\left[F\left(c_{1}\right) \cap H\left(c_{1}\right)\right] \vee\left[F\left(c_{2}\right) \cap H\left(c_{2}\right)\right]$ and $\left[F\left(c_{1}\right) \cap H\left(c_{1}\right)\right] \vee\left[F\left(c_{2}\right) \cap H\left(c_{2}\right)\right]$ exist in $(F, A) \sqcap_{E}(H, B)$.

Thus for all $e_{1}, e_{2} \in C=A \cup B$, we get $K\left(e_{1}\right) \vee K\left(e_{2}\right), K\left(e_{1}\right) \wedge K\left(e_{2}\right)$ exist in $(K, C)$. Therefore $(K, C)$ is a lattice ordered soft group.

\section{Conclusion}

In this paper, concept of lattice ordered soft group has been introduced. This work focused on operation of lattice ordered soft group. To extend this work, one can investigate the other (lattice based) algebraic properties.

\section{Acknowledgements}

Authors are highly thankful for nice comments and suggestions given by referees for improving this work. 
This research did not receive any specific grant from funding agencies in the public, commercial, or not-for-profit sectors.

\section{References}

[1] H. Aktas and N. Cagman, Soft sets and soft groups, Inform. Sciences, 177 (2007), 27262735 .

[2] Aslihan Sezgin, Akin Osman Atagun, On operations of soft sets, Computers and Mathematics with Applications, 61 (2011), 1457-1467.

[3] M.I. Ali, F. Feng, X.Y. Liu, W.K. Min, M. Shabir, On some new operations in soft set theory, Comput. Math. Appl., (2008), 2621-2628.

[4] M. I. Ali, A note on soft sets,rough sets and fuzzy sets, Soft Comput., 11 (2011), 332-3332.

[5] M. I. Ali, Tahir Mahmood, M. Muti Ur Rehman, M. Fahim Aslam, On lattice ordered soft sets, Applied Soft Computing, 36 (2015), 499-505.

[6] K. V. Babitha, J. J. Sunil, Soft set relations and functions, Comput. Math. Appl., 60 (2010), (1840-1849).

[7] Faruk Karaasalan, Cagman. N and Enginoglu. S, Soft lattices, J. New Res. Science (2012), 5-17.

[8] Fu Li, Soft lattices, Global Journal, 10, No. 4, (2010), 56-58.

[9] Fu Li, Notes on soft operations, APRN Journal, 6 (2011), 2222-9833.

[10] Garett Birkhoff, Lattice theory, American Mathematical Society Colloqium Publications, XXV.

[11] George Gratzer, Geneneral Lattice Theory, Second Edition, Birkhauser verlac, Boston, 1998.

[12] A. M. Ibrahim, A. O. Yusuf, Development of soft set theory, vol. 2. No. 9 (2012).

[13] V. D. Jobish, K. V. Babitha, Sunil Jacob John, On soft lattice operations, 5 issue 2, (2013), 71-86.

[14] P. K. Maji, R. Biswas, A. R. Roy, soft set theory, Comput. Math. Appl., 45 (2003), 555-562.

[15] D. Molodtsov, Soft set theory - First result, Comput. Math. Appl., 37 (1999), 19-31.

[16] Muhammad Aslam, Saqib Mazher Qurashi, Some Contributions to soft groups, Annals of Fuzzy Mathematics and Informatics, vol. 4, No. 1 (2012), 177-195.

[17] E. K. R. Nagarajan and Ganesamoorthi Meenambigai, An Application of Soft sets to Lattices, Kragujevac. Journal of Mathematics, vol. 35, No. 1 (2011), 75-87.

[18] E. K. R. Nagarajan and P. Geetha, Characterization Theorems for Modular and Distributive Soft Lattices, Intnl. Jorl. of Innovative Research in science Eng. and Tech., 2, issue 11 (2013), 2319-8753

[19] Natarajan. R and Vimala. J, Distributive l-ideal in a commutative l-group, Acta Ciencia Indica, 33 M. No. 2. 517 (2007)-MR 2392259.

[20] Natarajan. R and Vimala. J, Standard $l$-ideal in a commutative l-group, Acta Ciencia Indica, 33 M. No. 2. 539 (2007)-MR 2392261. 
[21] Onyeozili I. A. , Gwary T. M, A Study on the Fundamentals of Soft Set Theory, International Journal of Scientific and Technology Research, 3 issue 4 (2014), 132-143.

[22] Pei. D and Miao. D, From soft set to information systems, In:Proceedings, Granular computing, IEEE, Int. Conf., 2 (2005), 617-612.

[23] Xia Yin, Zuhua Liao, Study on soft groups, Journal of Comp., 8 (2013).

[24] Vimala. J, Neutral Operators and Triple Representation, Int. Jour. of Pure and Applied Mathematics, 91 issue 4 (2014), 477-481.

[25] Vimala. J and Bharathi. P, The Role of Fuzzy $l$-ideals in a Commutative $l$ - group, Global Journal of Pure and Applied Mathematics, 12 issue 3 (2016), 2067-2074.

[26] YingehaoShao, KeyunQin, The Lattice structure of the soft groups, Procedia Engineering, 15 (2011), 3621-3625. 
